keitswahlrecht innerhalb des Personalvertretungsrechts zu ermöglichen. Das Land Hessen ist hier, wie bei anderen Teilen seines Landesrechts (Quotierung zwischen Männern und Frauen), bereits den richtigen Weg gegangen.

\title{
8. Ergebnis
}

Die vorliegende Entscheidung stärkt die parteienstaatliche Komponente des öffentlichen Dienstes erheblich. Die These von der ausschließlichen Legitimation des öffentlichen Dienstes durch »Volk und Parlament « überdehnt deren Reichweite und unterschätzt die pluralistischen Systemelemente des modernen demokratischen Gemeinwesens ebenso wie die Legitimation aus Effektivität. Letztlich verkennt das BVerfG den Begriff der sozialen Demokratie. Daß das BVerfG seine dramatische Rechtsprechungskorrektur gegenüber BVerfGE 9, 268 in keiner Weise ausführlich darlegt, erhöht nicht die Akzeptanz des Judikats. Allerdings wird man einräumen müssen, daß das geltende Personalvertretungsrecht für den öffentlichen Dienst kein legislatorisches Glanzstück darstellte. Auch waren wichtige gewerkschaftliche Positionen keineswegs von Selbstwidersprüchen frei.

Gewerkschaftspolitisch ist die Frage zu stellen, ob es weiterhin sinnvoll ist, die umfassende Autonomie des öffentlichen Dienstes zu stützen oder ob nicht eine offensive Angleichung an die Verhältnisse der privatwirtschaftlich organisierten Bereiche anzustreben ist. Instrumente der zwingend notwendigen Modernisierung des öffentlichen Sektors können dabei Vereinbarungen zwischen Arbeitgebern und Gewerkschaften sein, die die Anliegen der laufenden Modernisierung und der öffentlichen Dienste aufnehmen und voranbringen, sowie ein verbessertes Personalratswahlrecht.

\section{Götz Frank \\ Der verfassungsrechtliche Rahmen zum sozialökologischen Jahr als Pflichtjahr ${ }^{\mathrm{r}}$}

Es gibt nur wenige verfassungsrechtliche Materien, die so stark von geschichtlichen Erfahrungen geprägt sind wie die in der Verfassung verankerten Grundpflichten. $\mathrm{Ob}$ sie Teil der demokratischen Tradition sind oder, ganz im Gegenteil, eher die nichtdemokratischen Epochen seit Beginn der demokratischen Entwicklung in Europa bestimmen, ist sogar im Hinblick auf die allgemeine Wehrpflicht lebhaft umstritten.

Die »levée en masse» von 1792 in Frankreich wird inbesondere vom konservativen

\footnotetext{
I Die verfassungspolıtische Diskussion um dıe Einfuhrung einer allgemeınen Dienstpflıcht geht quer durch die politischen Lager. In der CDU wurde ein dahingehender Antrag auf dem Bundesparteitag 1994 mit 290 gegen 236 Stimmen abgelehnt (s. Ekkehart/Lıppert, Allgemeıne Dienstpflicht als sıcherheits- und sozialpolitischer Ausweg? Vierteljahresschrift fur Sicherheit und Frieden, I 995, S. 72 ff., S. 72). Beı den Grunen wird enne dahingehende Forderung etwa von Daniel Cohn-Bendit vertreten. In der wissenschaftlichen Diskussion tritt Berthold Meyer (Hessische Stiftung Frıedens- und Konfliktforschung - HSFK -) fur ein mattraktıves Angebot frewwilliger Gesellschaftsdienste a anstelle von allgemeiner Dienstpflıcht und Wehrpflicht ein, ein Vorschlag, dem ich mich noch am ehesten anschließen konnte (In: Die bessere Alternative, Vierteljahresschrift fur Sicherheit und Frieden 1995, S. 79ff.). Zur Diskussion um die Abschaffung der Wehrpflicht s. meinen Bestrag "Der Aufgabenwandel der Bundeswehr und die Wehrpflicht", in: Fachzeitschrıft zur Kriegsdienstverweigerung, Wehrdienst und Zivildıenst, 1995, S. $140 \mathrm{ff}$.
} 
Staatsrecht gerne als Ausdruck der gewonnenen Volkssouveränität und des auf den Fahnen der Revolution stehenden Gleichheitsprinzips verstanden. ${ }^{2}$ In Wahrheit hat jedoch die französische Nationalversammlung von Anfang an die allgemeine Wehrpflicht grundsätzlich und entschieden abgelehnt, weil sie mit der Freiheit des Individuums unvereinbar sei. Allein das Werbesystem sei die einem freien Volk gemäße Rekrutierungsweise. Erst nach mehreren Niederlagen der französischen Freiwilligenverbände, die Frankreich an den Rand des Abgrunds brachten, setzte Carnot im Gegensatz zu den ursprünglichen Ideen der Revolution die allgemeine Wehrpflicht durch. ${ }^{3}$ Die Entscheidung des französischen Präsidenten im Februar 1996, die Wehrpflicht in Frankreich abzuschaffen, ist denn auch kein Bruch mit der französischen Tradition. Sie orientiert sich wieder an pragmatischen Gesichtspunkten, wie Kosten und Wandel der Aufgaben für die Armee. Die Verfassungstradition Frankreichs weist in ihrem demokratischen Beginn eine klare Tendenz gegen allgemeine Dienstpflicht auf. In Art. I 8 der Erklärung der Menschen- und Bürgerrechte der französischen Verfassung von 1793 hieß es unter anderem "Das Gesetz erkennt keine Dienstbarkeit an; nur über die Dienstleistungen und Entschädigungen dafür kann zwischen dem Menschen, der arbeitet, und dem, der ihn anstellt, eine Vereinbarung stattfinden $\ll .^{4}$

Ein Jahr, nachdem die Wehrpflicht in der französischen Revolution eingeführt war, wurde von Therese Cabarrus-Fontenay die Forderung nach einem Pflichtjahr erhoben. Vor dem Hintergrund der nach wie vor primär vom Freiheitsgedanken durchdrungenen Verfassungsentwicklung konnte sich diese Forderung in Frankreich jedoch nicht durchsetzen. Die ältere westliche Demokratie als die französische, die amerikanische, hielt von der allgemeinen Wehrpflicht gar nichts und hat sie nur während der beiden Weltkriege eingeführt und nach dem Vietnamkrieg wieder abgeschafft.' Demgegenüber führte man in Preußen I814, zu einem Zeitpunkt also, zu dem man von einer demokratischen Verfassungstradition weit entfernt war, wegen der katastrophalen Niederlagen des alten friderizianischen Heeres gegen Napoleon im Zuge der Scharnhorstschen Heeresform die allgemeine Wehrpflicht ein. ${ }^{6}$

Dic Idee eines Arbeitsdienstes, allerdings auf freiwilliger Basis, tauchte dann in Frankreich I 848/49 in Form der sogenannten Nationalwerkstätten auf Betreiben des Sozialisten Louis Blanc wieder auf. Louis Blanc ging es allerdings darum, mit diesen Nationalwerkstätten den privatkapitalistischen Betreibern Konkurrenz zu machen und das von ihm propagierte Recht auf Arbeit zu verwirklichen. An diese Idee knüpfte Ferdinand Lasalle in den soer Jahren an, als er "Produktivassoziationen mit Staatshilfe « forderte. ${ }^{7}$

In Deutschland kam es dann während des Kaiserreichs verschiedentlich zu Forderungen nach Einführung einer Arbeitspflicht, wobei die prominenteste Vertreterin 1915 Helene Lange war, als sie auf der Pfingsttagung des Allgemeinen Deutschen Lehrerinnenvereins »die weibliche Dienstpflicht in wihrer wesentlichen Bestimmung in den dauernden Friedensaufgaben « ansah, worunter sie vor allen Dingen "Krankenpflege und aller Art organisierter Wohlfahrtspflege « ansah. Durchsetzen konnte sich aber auch im Ersten Weltkrieg nur ein freiwilliger weiblicher Hilfsdienst,

2 So insbesondere von der Heydte, Wehrpflicht, in: Staatslexikon der Gorres-Gesellschaft, Band 8, 1963, S. 494 sowie Hans Seider/Helmut Reindel, Die Wehrpflicht, Wien 1971, S. 36.

3 Christian Grimm, Allgemeine Wehrpflicht und Menschenwurde, Berlin 1982, S. 19f.

4 Zitiert nach der Ubersetzung von G. Franz, Staatsverfassungen, 3. Aufl., 1975, S. 311

s Vgl. Manfred Messerschmidt, Der Staatsbürger muß sich nıcht uber die Uniform legitimieren, In: Antimilitarısmus-Information 1994, Heft 10, S. 8 ff.

6 Siehe Grimm, (Fn. 3), S. 20.

7 Wolfgang Wippermann, Arbeitsdienst im Dritten Reich, in: Antımilitarismus-Information, 1994, S. ${ }_{4} \mathrm{ff}$. S. Is. 
ganz im Gegensatz zu den gesetzlichen männlichen Hilfsdienstpflichten aufgrund des Gesetzes über den vaterländischen Hilfsdienst. ${ }^{8}$

Erstmals in einen Verfassungstext aufgenommen wurde die Arbeitspflicht am Io. Juli 1918, allerdings nicht in einem demokratischen Staat, sondern in der Russischen Sozialistischen Föderativen Sowjetrepublik, in deren Verfassung es in Art. I 8 hieß: "Die Russische Sozialistische Föderative Sowjetrepublik erklärt die Arbeit zur Pflicht aller Bürger der Republik und verkündet die Losung: , Wer nicht arbeitet, soll auch nicht essen «. ${ }^{9}$ Dieser Verfassungstext hatte als Vorbild für die sozialistischen Staaten seine Funktion, etwa auch für die Verfassungsgeschichte der DDR, in der es ebenfalls die Arbeitspflicht als Pendant zu den Rechten der Staatsbürger gab. ${ }^{10}$

In den Beratungen zur Weimarer Reichsverfassung spielte zumindest in der verfassunggebenden Nationalversammlung diese Entwicklung in der Sowjetrepublik durchaus eine Rolle. ${ }^{11}$ Gleichwohl kam es in der Weimarer Reichsverfassung zu keinen vergleichbaren Grundverpflichtungen. Art. I 32 Weimarer Reichsverfassung enthielt die Verpflichtung zur Übernahme ehrenamtlicher Tätigkeiten nach Maßgabe der Gesetze. In den ehrenamtlichen Tätigkeiten sah man damals wie heute das politische Selbstverwaltungsprinzip verwirklicht, nicht aber eine Grundlage für Grundpflichten im eigentlichen Sinne, da hier der Bürger ja selbst in eine organschaftliche Tätigkeit des Staates eingebunden werden sollte, somit in eigener Person Staatlichkeit mit verkörpern sollte. ${ }^{12}$ Ehrenämter unterfallen deswegen auch heute nicht dem $\mathrm{Ar}$ beitsbegriff des Art. 12 Abs. 2 GG. Art. 163 Weimarer Reichsverfassung enthielt die sittliche Pflicht des Bürgers, seine geistigen und körperlichen Kräfte so zu betätigen, wie es das Wohl der Gesamtheit erfordere. Dies war eine sittliche und eben gerade keine Rechtspflicht zur Arbeit. ${ }^{13}$ Art. 133 Weimarer Reichsverfassung enthielt die Verpflichtung, nach Maßgabe der Gesetze persönliche Dienste für den Staat und die Gemeinde zu leisten. Die Rechtspraxis der Weimarer Zeit konkretisierte diesen Artikel ähnlich wie dies heute im Hinblick auf Art. I 2 Abs. 2 GG geschieht; gemeint waren damit in erster Linie polizeiliche und kommunale Leistungspflichten, wie die Hand- und Spanndienste, die Feuerwehrpflicht und die Nothilfepflichten, nicht aber darüber hinausgehende Dienstverpflichtungen. ${ }^{14}$ Ich werde auf diese Begriffe noch bei der Darstellung der Auslegung zu Art. 12 Abs. 2 GG zurückkommen.

So blieb es in der deutschen Rechtsgeschichte seit dem Beginn der demokratischen Entwicklung neben der erwähnten Regelung in der DDR-Verfassung bei der einzigen Ausnahme des nationalsozialistischen Staates durch das Reichsarbeitsdienstgesetz vom 26. Juli 1935. ${ }^{\text {'s }}$ Dieses Reichsarbeitsdienstgesetz hat auf der einen Seite damit zu tun, daß seit Beginn des Autobahnprogramms die Rüstungsindustrie um knapper werdende Arbeitskräfte besorgt war. Die Ausgaben für die Wehrmacht hatten sich vom Zeitpunkt der Machtübernahme in zwei Jahren vervierfacht und stiegen bis 1938 im Vergleich zu 1933 auf das Zehnfache an. Trotz der zu Beginn der nationalsozialistischen Zeit bestehenden Arbeitslosigkeit ist aus diesen Zahlen er-

8 Nachweise siehe Klaus Kübler, Das Pflichtjahr fur Madchen, DÓV 1962, S. 809 ff., S. 810.

9 Zitiert nach Volkmar Götz, Grundpflichten als verfassungsrechtliche Dımension, VVDStRL 4 1, S. 7 ff., S. 19.

10 Siehe nảher hierzu Gotz, (Fn. 9), S. 20

I I Vgl. den dahingehenden Hinweis von Götz, (Fn. 9), S. I 9, mit dem Hinweis auf die Einfuhrungsrede zur Beratung der Grundrechte von Friedrich Naumann (FN 54).

12 Vgl. hierzu Monika Gunther, Die Heranziehung als Volkszahler, DVBl 1988, S. 429 ff., S.429.

13 So zu Recht Jost Pietzcker, Gutachten zu Rechtsfragen der Einfuhrung einer allgemeinen Dienstleistungspflicht, Gutachten erstellt für den Gesetzgebungs- und Beratungsdienst des Deutschen Bundestages im Oktober 199x (unveroffentlicht), S. 25.

I4 Anschütz, Die Verfassung des Deutschen Reiches, 14. Aufl., 1933, Art. 133, Anmerkung 1, S. 6 Is

is Reichsgesetzblatt I, 1935, S. 769 . 
sichtlich, daß es zu einem Arbeitskräftemangel kam und kommen mußte. ${ }^{16}$ Vor allem diente aber der Reichsarbeitsdienst der ideologischen, insbesondere rassistischen und militaristischen Indoktrinierung, der politischen Disziplinierung und letztlich der Vorbereitung auf den Kriegsdienst. ${ }^{17}$ Dies kommt in $\mathbb{S}$ I Abs. 3 des Reichsarbeitsdienstgesetzes auch deutlich zum Ausdruck, in dem es hieß: "Der Reichsarbeitsdienst soll die deutsche Jugend im Geiste des Nationalsozialismus zur Volksgemeinschaft und zur wahren Arbeitsauffassung, vor allem zur gebührenden Achtung der Handarbeit erziehen «.

Die Entstehungsgeschichte des Art. I 2 Abs. 2 GG ist als eine bewußte Abkehr von der nationalsozialistischen Indienstnahme der Arbeitskraft des Einzelnen für den Staat zu verstehen. Das Bundesverfassungsgericht hat dies immer wieder betont und zugleich darauf hingewiesen, daß das westliche Freiheitsverständnis gegenüber dem in kommunistisch beherrschten Gebieten damals auch im Bereich der Arbeit angewandten Zwangsmaßnahmen unterstrichen werden sollte. ${ }^{18}$ Im Parlamentarischen Rat entstand, wie schon in der vorangegangenen Debatte in Herrenchiemsee, die Konzeption des Art. I 2 Abs. 2 GG im Sinne einer Antwort des demokratischen Neubeginns. Zugleich standen freilich die Mitglieder des Parlamentarischen Rats unter dem Eindruck der Dienstverpflichtungen in der Besatzungszeit, die ebenfalls ausgeschlossen werden sollten. Deswegen wurden als unzulässige Dienstverpflichtungen auch Beispiele erörtert, die sich sozusagen vor den Augen des Parlamentarischen Rates abspielten, wie Arbeitszwang in der amerikanisch besetzten Zone, Schutträumverpflichtungen in britisch besetzten Städten, Uranabbau, Schippverpflichtung von Arbeitslosen unter Androhung des Entzugs der Arbeitslosenunterstützung und ähnlichem. ${ }^{19}$

Das Bundesverfassungsgericht hat diese Erfahrung aus der Genese des Art. 22 Abs. 2 GG für die Auslegung als nach wie vor im Zentrum stehende Überlegung unterstrichen. Die Bedeutung und Tragweite dieser Verfassungsvorschrift lasse sich nicht allein vom gängigen Wortsinn her erfassen; vielmehr sei der Blick auf das rechtliche und historische Umfeld der Entstehung der Verfassungsbestimmung, wie sie sich in den Beratungen dargestellt habe, maßgeblich. ${ }^{20}$ Der Bundesverfassungsrichter Dieter Grimm hat auf der Tagung der Staatsrechtslehrer in Konstanz 1982, als über die Grundpflichten als verfassungsrechtliche Dimension debattiert wurde, ganz wesentliche Aspekte der freiheitlich-demokratischen Tradition herausgestellt, als er ausführte, der Grund für Grundrechte läge in der überlegenen Macht des Staates; ein vergleichbarer Grund für Grundpflichten sei hingegen nicht erkennbar. ${ }^{21}$ Die Geschichte der Arbeitspflichten in der freiheitlichen Demokratie belegt gerade in Deutschland, daß man sich hier trotz immer wieder erhobener Forderungen mit der verfassungsrechtlichen Verankerung schwer tat und eher nach der klaren Begrenzung von Grundpflichten suchte, um dem Staat nicht noch einmal die Möglichkeit einzuräumen, mit solch einem Instrument Mißbrauch zu betreiben.

Vor dem Hintergrund dieser von der historischen Auslegungsmethode und den geschichtlichen Erfahrungen bestimmten Interpretation kommt die herrschende Lehre

I6 Naher hierzu siehe Wippermann (Fn. 7), und Hans-Ulrich Thamer, Verfuhrung und Gewalt. Die Deutschen und ihre Nation, Berlin 1986, insbesondere S. 476 ff. und im Hinblick auf die totale Mobilmachung: Die Mobilmachung der deutschen Frauen, S. s16ff. (siehe dazu die Verordnung uber die Durchführung der Reichsarbeitsdienstpflicht fur die weibliche Jugend vom 4. 9. 1939, Reichsgesetzblatt I, Nr. 170, S. 1857 )

17 So zu Recht Wippermann (Fn. 7), S. i7.

18 BVerfGE 74, 102, 1 16, anknupfend an BVerfGE 22, 380, 383

19 Vgl. hierzu Pietzcker (Fn. 13), S. 6 f. mit nảheren Hinweisen.

20 BVerfG NJW 1991, S. $1043,1044$.

21 VVDStRL $_{41}$, S. $102 \mathrm{f}$. 
zu dem Ergebnis, daß die Einführung eines sozialökologischen Jahres als Pflichtjahr auf der Grundlage des vorhandenen Verfassungstextes nicht realisierbar ist. Wenn die politischen Parteien im Bundestag dies wirklich wollen, so müssen sie sich zu einer Verfassungsänderung entschließen und dafür auch die notwendige 2/3-Mehrheit aufbringen.

Im einzelnen ergibt sich dies vor allem aus Art. 12 Abs. 2 GG und aus Art. I2a GG. Art. 12 Abs. 2 GG enthält mit der Formulierung » Niemand darf zu einer bestimmten Arbeit gezwungen werden « ein Grundrecht auf Freiheit von Arbeitszwang. ${ }^{22}$ Verboten ist danach jede mit $Z$ wangsmitteln durchsetzbare hoheitliche Heranziehung zu einer bestimmten Arbeit. ${ }^{23}$ Der Begriff $»$ Arbeit wird nicht akzeptiert, wenn der Bürger seiner Meldepflicht nachkommt, der Kaufmann seiner Buchführungspflicht genügt, der Eigentümer seine Verkehrssicherungspflicht erfüllt oder aber, wenn es sich um Pflichten handelt, die dem Einzelnen um seiner selbst willen auferlegt werden, wie die gesetzliche Gesundheitskontrolle, die Schul- oder Ausbildungspflicht. ${ }^{24}$ Kurzum, wenn es sich um höchstpersönliche Pflichten, Nebenpflichten, die aus einer anderen Rechtsposition abgeleitet werden oder aber Pflichten handelt, die wie die Schulpflicht als Teil "kompensatorischer staatlicher Fürsorge «"s gelten kann, ist nicht von Arbeit im Sinne des Grundgesetzes zu sprechen. Um Arbeit handelt es sich aber zweifellos beim sozialökologischen Pflichtjahr.

Das Abwehrrecht des Art. 12 Abs. 2 GG gegen staatlichen Zwang zu einer bestimmten Arbeit unterliegt allerdings Schranken. Im Rahmen einer herkömmlichen allgemeinen, für alle gleichen öffentlichen Dienstleistungspflicht sind für staatliche Dienstpflichten Möglichkeiten eröffnet. Allgemein ist die Dienstleistungspflicht, wenn sie grundsätzlich jedermann trifft, und gleich ist sie, wenn sie allen Betroffenen ohne sachlichen Unterschied auferlegt wird.

Das zentrale Kriterium für die Konkretisierung der Schranken des Art. 12 Abs. 2 GG ist freilich der Begriff der Herkömmlichkeit. Herkömmlich ist eine Dienstpflicht, wenn sie nicht unerhebliche Zeit bestanden hat und im Rechtsbewußtsein als überkommener Bestandteil der Pflichtenordnung verwurzelt ist. ${ }^{26}$ Die sogenannten Hand- und Spanndienste im landwirtschaftlichen Bereich, die insbesondere für den Wegebau galten, können heute nicht mehr als herkömmlich gelten, da sie durch den modernen Straßenbau und die Gebiets- und Gemeindereformen in den 7oer Jahren als überholt gelten müssen. ${ }^{27}$ Erwähnt werden insbesondere für die süddeutschen Staaten, in denen entsprechende Regelungen in den Landesverfassungen verankert sind, nach wie vor die Feuerwehr, die Wasser- und Deichwehrpflicht, die Straßenreinigungs-, die Räum- und Streupflicht, die Katastrophenschutzpflicht sowie die

22 Unstreitug vgl. Pietzcker (Fn. 13), S. 9.

23 AKGG Rittstieg, 2. Aufl., Art. 12, Rz. 160.

24 Siehe Scholz, in: Maunz/Durig, Kommentar zum Grundgesetz, Art. 12 Rz. 48 I. Nach der standigen Rechtsprechung des Bundesverwaltungsgerıchts liegt ebenfalls kein Verstoß gegen die Verbote des Arbeitszwangs und der Zwangsarbeit vor, wenn Sozialhilfeleistungen von Leistung zumutbarer sogenannter GZ-Arbeit seitens des Hilfesuchenden abhang1g gemacht werden. Vgl. etwa BVerwGE 11,252 ; 12 , 132 ; 29, 99. Auf die verfassungsrechtlichen Bedenken, die gegenuber dieser Praxis erhoben werden kónnen, weist zu Recht Dieter Sterzel in seinem Beitrag Zwang zur Arbeit im Sozialstaat, in: Festschrift fur Helmut Ridder zum 70. Geburtstag, Neuwied/Frankfurt 1989, S. 269 ff., hin.

25 So die Umschreibung von Hofmann, Grundpflichten als verfassungsrechtliche Dimension, VVDStRL 4 I, S. 66 .

26 Scholz (Fn. 24), Rz.486. Diese Dienstpflichten galten als im Interesse des Allgemeinwohls notwendige Arbeiten, wobei sich dies aus dem Aufgabenzweck, nicht aber den allgemeinen Haushaltsentlassungseffekten ergab (naher hierzu: Uwe Berlit, Verpflichtung zur Selbsthilfe, Verbot zur Zwangsarbeit, RsDE Heft 33 (1996), 29ff., 61 f.).

27 So im Ergebnis auch Gotz (Fn. 9), S. 28 und Christoph Gusy, Arbeitszwang - Zwangsarbett - Strafyollzug - BVerfGE 74, 102, JuS 1989, S. 102 ff., S. 713. 
Hilfspflicht bei Unglücksfällen. ${ }^{28}$ Das sozialökologische Pflichtjahr scheitert am Begriff der Herkömmlichkeit, weil es während keiner verfassungsrechtlich vergleichbaren Epoche bestand. Es wäre absurd, hiergegen mit der Zeit des Nationalsozialismus und dem da begründeten Reichsarbeitsdienst zu argumentieren, da es sich hier in der deutschen Verfassungsgeschichte um eine Ausnahmeerscheinung handelte, die aus dem Rahmen der verfassungsgeschichtlichen Tradition deutlich herausfällt. Dies widerspräche auch der Intention der Verfassungsväter, die gerade in diesem Punkt gegen eine Wiederholung nationalsozialistischer Gebräuche wirksame Sperren aufbauen wollten. ${ }^{29}$

Eine spezielle Regelung, die der Vorschrift des Art. I 2 Abs. 2 GG gegenüber vorgeht, hat der Verfassunggeber im Hinblick auf die Wehr- und Ersatzdienstpflicht getroffen. Die nach Art. 12a Abs. I GG in einer Kannvorschrift begründete Wehrpflicht kann in den Streitkräften, im Bundesgrenzschutz oder in einem Zivilschutzverband abgeleistet werden. Nach Art. I 2a Abs. 2 GG kann im Falle der Verweigerung aus Gewissensgründen zu einem Ersatzdienst verpflichtet werden. Diese Spezialpflichten im Verhältnis zu Art. 12 Abs. 2 GG lassen sich aber nicht erweiternd dahin auslegen, daß sie dem Gesetzgeber die Möglichkeit einräumen würden, aufgrund des durch sie eröffneten Rahmens ein sozialökologisches Pflichtjahr einzuführen. Die in den Streitkräften oder im Bundesgrenzschutz begründeten Pflichten lassen dies schon vom Wortlaut her nicht zu. Auch die Pflichten im Zivilschutz müssen schon wegen ihres systematischen Zusammenhangs zu den genannten Pflichten als verteidigungsbezogen angesehen werden. ${ }^{30}$

Erweiterungsmöglichkeiten lassen sich aus Art. I2a GG allenfalls aus dem in Abs. 2 angesprochenen Ersatzdienst ableiten. Der Ersatzdienst, wenngleich er gemeinschaftsnützlich angelegt ist, ist aber nicht um seiner selbst willen eingerichtet worden. Sonst wäre es kaum zu rechtfertigen, daß Frauen zu diesem Dienst nicht verpflichtet sind. Er ist vielmehr aus der Wehrpflicht als Surrogat abgeleitet worden und wäre ohne diese verfassungsrechtlich nicht begründbar. ${ }^{31}$ In Zeiten wie heute, in denen wegen der zahlenmäßigen Verringerung der Bundeswehr nach der Schaffung der deutschen Einheit darüber nachgedacht wird, ob man nicht zu einem Freiwilligenheer übergehen könne, ist es ohne Zweifel nach der Verfassungslage nicht möglich, anstelle dessen über neue Pflichten in einem sozialökologischen Pflichtjahr nachzudenken, die an die Stelle einer abgeschafften oder zumindest wesentlich verringerten Wehrpflicht treten könnten. Wer dies will, muß die Verfassung auch in ihren Aussagen in Art. г 2a Abs. I und Abs. 2 GG ändern.

An diesem Ergebnis aufgrund der verfassungsrechtlichen Aussagen von Art. 12 Abs. 2 und Art. x 2a GG ändert sich auch nichts, wenn man auf den Gleichheitsgrundsatz des Art. 3 GG und eine etwaige Ungleichbehandlung von Männern und Frauen hinweisen wollte. Ohnehin erscheint dies schon deswegen fragwürdig, weil es neben der Landesverteidigung in unserer Gesellschaft auch andere Aufgaben gibt, die vorrangig von einer der beiden Gruppen erbracht werden. Man denke nur an die nach wie vor primär in Händen der Frauen liegende Kindererziehung. ${ }^{32}$ Auch ohne diese Überlegungen wäre eine solche Folgerung aber schon aus verfassungssystema-

28 Art. 122 Bayerische Verfassung; Art. 1o Bremische Verfassung; Art. 22 Rhennland-Pfalzische Verfassung sowie Art. 19 Saarlandische Verfassung, naher hierzu siehe Gusy (Fn. 27), S. 713.

29 Unstreitig vgl. etwa Gusy (Fn. 27), Kubler (Fn. 8), S. 812 und Pietzcker (Fn. 13) mıt weiteren Nachweisen.

30 So zu Recht P1etzcker gegen Scholz, in: Maunz/Durig, Kommentar zum Grundgesetz, Art. I2a, Rz. 63 .

31 So auch Gotz (Fn.9), s. 25 f.

32 Vgl. Walburga von Zammek/Dorothea Schafer, Soziales Pflichtjahr fur junge Frauen?, Sozialer Fortschritt 1989 , Heft I, S. 17 ff., S. 18. 
tischen Gründen nicht zulässig. Die Regelungen des Art. I 2 Abs. 2 und I za GG haben gleichen Rang zu den verfassungsrechtlichen Bestimmungen der Art. 3 Abs. 2 und 3 GG und werden, wie dies die Rechtsprechung des Bundesverfassungsgerichts auch klar zum Ausdruck gebracht hat, durch die allgemeine Überlegung zur Gleichbehandlung nicht relativiert. ${ }^{33}$

Ebensowenig erscheint es möglich, Arbeitspflichten, die vom Staat begründet werden, auf das Sozialstaatsprinzip zu stützen. Zweifellos verkörpert das Sozialstaatsprinzip auch den Gedanken der Solidarität, so daß man versucht sein könnte, dies bei der Verpflichtung zu gemeinwohlorientierter Arbeit mit heranzuziehen. Selbst wenn der Solidaritätsgedanke solche Ansätze böte, wäre aber für das Sozialstaatsprinzip in erster Linie doch immer auf die Spezialregelung des Verfassunggebers in Art. I 2 Abs. 2 und i 2 a GG zu verweisen, die dem allgemeinen Grundsatz vorgehen. Der Begriff der Solidarität ist zudem gerade kein Begriff, der zu staatlichen Eingriffen in den bürgerlichen Freiheitsraum ermächtigt. Er muß vielmehr dem Verhältnis der Bürger zueinander vorbehalten bleiben. ${ }^{34}$ Deswegen kann Solidarität allenfalls als Argument für die Schaffung verfassungsrechtlicher Grundpflichten, nicht aber als verfassungsrechtliche Grundlage für gesetzliche Grundpflichten angesehen werden, wenn sie der Verfassunggeber selbst noch nicht ausdrücklich anerkannt hat. ${ }^{35}$

Im Ergebnis komme ich also dazu, daß die Einführung eines sozialökologischen Pflichtjahres ohne Verfassungsänderung nicht zu bewerkstelligen sein wird. Die Verfassungsänderung halte ich allerdings aus verfassungsrechtlicher Sicht für möglich. Sie scheitert nicht an Art. 79 Abs. 3 GG, dem sogenannten verfassungsfesten Minimum, weil sie gegen die Menschenwürde verstößt. ${ }^{36}$ Das Bundesverfassungsgericht differenziert sorgfältig zwischen Zwangs- und Pflichtarbeiten als Methode der Rekrutierung und Verwendung von Arbeitskräften für Zwecke der wirtschaftlichen Entwicklung als solcher auf der einen Seite und erzwungenen Arbeiten, die die Menschenwürde mißachten, auf der anderen Seite. ${ }^{37}$ Beide fallen in den Schutzbereich des Art. 12 Abs. 2 und 3 GG, aber nur die letztere zugleich in den des Art. I GG. Arbeitsdienstpflichten auf dem Level des Reichsarbeitsdienstes würden bei einer Verfassungsänderung am verfassungsfesten Minimum der Menschenwürde scheitern; unterhalb dessen sind aber auch Lösungen denkbar, die nicht am Verdikt des Art. 79 Abs. 3 GG scheitern. ${ }^{38}$

Auch die allgemeinen Regeln des Völkerrechts können den deutschen Verfassunggeber nicht an einer Verfassungsänderung in diesem Sinne hindern. Nach Art. 25 GG sind sie zwar Bestandteil des Bundesrechts, aber im Rang unter den Bestimmungen des Grundgesetzes. ${ }^{39}$ Nach dem Vorbild des Übereinkommens über Zwangs- und Pflichtarbeit der Internationalen Arbeitsorganisationen von 1930 wurden sowohl in der Konvention zum Schutz der Menschenrechte und Grundfreiheiten als auch in dem Internationalen Pakt über bürgerliche und politische Rechte Verbote der Pflichtarbeit verankert, die allerdings unter anderem dann nicht gelten, wenn es sich um Dienstleistungen handelt, die zu den normalen Bürgerpflichten gehören. ${ }^{\circ}$ Nor-

33 Siehe BVerfGE I2, 45, $52 \mathrm{f}$.

34 AKGG Kittner, Art. 20 Abs. 1-3 IV, Rz.75.

35 So im Ergebnis auch Bachof, VVDStRL 41,126

36 In diese Richtung tendiert dagegen der Beitrag von Wolfgang Kaleck zur Verfassungsmaßıgkeit einer allgemeinen Dienstpflicht, in: Antimilitarismus-Informationen 10/94, S. 46 ff., S. 49 f. 37 BVerfGE 74, 102, $121 \mathrm{ff}$.

38 So im Ergebnis auch Pietzcker (Fn. 13), S. $36 \mathrm{f}$.

39 BVerfGE $37,271,279$.

$40 \mathrm{Vgl}$. Art. I des Ubereinkommens uber Zwangs- und Pflichtarbeit der Internationalen Arbeitsorganisationen ILO, Ubereinkommen Nr. 29 vom 28. Julı 1930 (BGBl. I956 II, S.64 I, 1963 II, S. 1 136), Art. 4 der Konvention zum Schutz der Menschenrechte und Grundfreiheiten vom 4.11.1950 (BGBl. 1952 II, S.685, 953) mit den dazu ergangenen Novellierungen, zuletzt BGBl. 1989 II, S. 991 sowie Art. 8 des 
male Bürgerpflichten können sicherlich nicht gleichgesetzt werden mit herkömmlichen Bürgerpflichten, aber es ist auch nicht möglich, ständig neue »normale Bürgerpflichten " zu kreieren..$^{41}$ Deswegen ist ein Verstoß gegen die internationalen Verträge bei der Einführung eines sozialökologischen Pflichtjahres denkbar. Diese sind freilich auch kündbar.

Sollte sich der Verfassunggeber in der Bundesrepublik wirklich mit dem Gedanken tragen, eine neue Grundpflicht einzuführen, so sollte er dabei den Ausnahmecharakter bedenken, den Grundpflichten in der demokratischen Tradition immer gehabt haben. Erweiterungen der verfassungsrechtlichen Grundpflichten stellen gerade für die deutsche Tradition ein verfassungspolitisches Thema von äußerster Brisanz dar. Es spricht viel dafür, daß man, bevor man an die Einführung einer neuen Verpflichtung durch das sozialökologische Jahr denkt, alle Möglichkeiten ausschöpft, die das freiwillige ökologische Jahr bietet..$^{42}$

\section{Ludwig Renck \\ Das Glaubensbekenntnis der Bundesverfassungsrichter}

Mit seinem auch sonst bemerkenswerten Beitrag in BayVBl. 1996, $107 \mathrm{ff}$. hat der Lt. Ministerialrat Dr. Eberl vom Bayerischen Kultusministerium einen Vorschlag in die Diskussion um den Schulkreuz-Beschluß des Bundesverfassungsgerichts eingebracht, der zu weiterführendem Nachdenken zwingt. Er meint, um religiöse Katastrophen bei der Rechtsanwendung zu vermeiden, sei zu empfehlen, die Bundesverfassungsrichter in Fällen, »in denen die Weltanschauung der Richter eine ausschlaggebende Rolle (sic!) spielen kann«, sich zu Verfahrensbeginn mit ihrem jeweiligen Bekenntnis outen zu lassen, da einerseits ihre politische Einstellung ohnehin bereits bekannt, andererseits von dem Glaubensbekenntnis eine verbesserte Entscheidungsqualität zu erwarten ist. Der Kundige bemerkt sofort, daß die Verhältnisse am Bundesverfassungsgericht offenbar mit denen in den Führungsriegen des Bayerischen Kultusministeriums verglichen werden können. Er fragt sich deshalb besorgt, warum bei religionsträchtigen Maßnahmen dieses Hauses, wenn und soweit es sie schon gibt, die ministeriellen Entscheidungsträger ihre jeweilige Glaubensüberzeugung für sich behalten sollten.

Werden etwa wieder einmal gegen den ausdrücklichen Spruch des Bundesverfassungsgerichts kirchliche Leitlinien als verbindliche Verwaltungsvorschrift für den allgemeinen Schulbetrieb im Ministerialamtsblatt veröffentlicht, so dürfte es dem Verständnis der Leserschaft durchaus entgegenkommen, wenn dort statt bisher schlicht »gez. NN, Ministerialrat« künftig deutlich "gez. NN, Ministerialrat (katholisch)« oder so ähnlich geschrieben steht. Dies würde zudem die Aussagekraft der Religionsstatistik erweitern, denn es bestünde die Möglichkeit, Verfassungswidrigkeiten dann auch konfessionell zu katalogisieren. Nicht einzusehen ist des weiteren,

Internationalen Pakts uber burgerliche und politische Rechte vom 19. 12.1966, BGBI. 1973, II, S. 1534 .

4 I Pletzcker (Fn. 13), S. 5

421994 gab es fur das freiwillige okologische Jahr pro Stelle zehn Bewerber. Die meisten Bundeslander tun sich schwer, in nennenswertem Umfang Stellen dafur einzurichten, und dıe Träger sehen sich finanziell uberfordert. Siehe Okologische Briefe Nr. 43 vom 26. 10. 1994, S. 3 ff. 